\title{
Bilinear Minimax Optimal Control Problems for a von Kárman System with Long Memory
}

\author{
Jin-soo Hwang $(\mathbb{D}$ \\ Department of Mathematics Education, College of Education, Daegu University, Jillyang, Gyeongsan, Gyeongbuk, Republic of Korea
}

Correspondence should be addressed to Jin-soo Hwang; jshwang@daegu.ac.kr

Received 18 February 2020; Accepted 12 May 2020; Published 16 June 2020

Academic Editor: Gisele Mophou

Copyright (C) 2020 Jin-soo Hwang. This is an open access article distributed under the Creative Commons Attribution License, which permits unrestricted use, distribution, and reproduction in any medium, provided the original work is properly cited.

\begin{abstract}
In the present article, we consider a von Kárman equation with long memory. The goal is to study a quadratic cost minimax optimal control problems for the control system governed by the equation. First, we show that the solution map is continuous under a weak assumption on the data. Then, we formulate the minimax optimal control problem. We show the first and twice Fréchet differentiabilities of the nonlinear solution map from a bilinear input term to the weak solution of the equation. With the Fréchet differentiabilities of the control to solution mapping, we prove the uniqueness and existence of an optimal pair and find its necessary optimality condition.
\end{abstract}

\section{Introduction}

Let $\Omega$ be an open bounded domain in $R^{2}$ with a sufficiently smooth boundary $\partial \Omega$. We set $Q=(0, T) \times \Omega, \Sigma=(0, T) \times \partial$ $\Omega$. We consider the following von Kárman system with long memory and the hinged boundary condition in the variables $u$ and $v$, representing the deflection of the plate and the Airy's stress, respectively:

$$
\left\{\begin{array}{l}
u^{\prime \prime}-\gamma \Delta u^{\prime \prime}+\Delta^{2} u+\int_{0}^{t} k(t-s) \Delta^{2} u(s) d s=[u, v]+q u+f \quad \text { in } Q, \\
\Delta^{2} v=-[u, u] \quad \text { in } \quad Q, \\
u=\Delta u=v=\frac{\partial v}{\partial v}=0 \quad \text { on } \quad \Sigma, \\
u(0, x)=u_{0}(x), \quad u^{\prime}(0, x)=u_{1}(x) \quad \text { in } \quad \Omega,
\end{array}\right.
$$

where ${ }^{\prime}=\partial / \partial t$, the vector $v$ denotes an outward normal, $\gamma>0$ means a constant related to the rotational inertia, $k(\cdot) \in C^{1}([0, T])$ is a memory kernel, $f$ is a forcing function, and $[\because, \cdot]$ is the von Kárman bracket given by

$$
[v, o]=\frac{\partial^{2} v}{\partial x_{1}^{2}} \frac{\partial^{2} o}{\partial x_{2}^{2}}+\frac{\partial^{2} o}{\partial x_{1}^{2}} \frac{\partial^{2} v}{\partial x_{2}^{2}}-2 \frac{\partial^{2} v}{\partial x_{1} \partial x_{2}} \frac{\partial^{2} o}{\partial x_{1} \partial x_{2}} .
$$

The term $q u$ in Equation (1) represents the reset force of the elastic plate in the system. This physical situation naturally leads to the consideration of the bilinear control problem for the control function $q$, which is used as a force to make the state close to a desired state taking into account. In this motivation, Bradley and Lenhart [1] studied the bilinear optimal control problem for a Kirchhoff plate equation (cf. [2]). And it has been studied in [3] the bilinear optimal control problem of velocity term in a Kirchhoff plate equation.

Motivated by $[1,3]$ with the above physical background, we study here the bilinear minimax control problem for Equation (1) with the control function $q$ based on the Fréchet differentiabilities of the nonlinear solution map. More detailed explanations are given as follows:

In our previous study [4], we considered the Dirichlet boundary value problems of Equation (1) without the term $q u$ and studied the optimal control problems for the external forcing control system by the frameworks in Lions [5]. In [4], we proved and used the Gâteaux differentiability of the nonlinear solution map to present the necessary optimality conditions for the optimal controls of the specific observation cases.

In this paper, we show the Fréchet differentiability of the solution map $q \longrightarrow u$ from the bilinear control input terms to 
the solutions of Equation (1). In most cases, the Gâteaux differentiability may be enough to solve a quadratic cost optimal control problem. However, the Fréchet differentiability of a solution map is more desirable for studying the problem with more general cost function like nonquadratic or nonconvex functions. So, this study is an improvement on a previous study [4]. Based on the result, we constructed and solved the bilinear minimax optimal control problems in Equation (1). The minimax control strategies have been used by many researchers for various control problems (see Lasiecka and Triggiani [6] and Li and Yong [7]). As explained in [8], the minimax control framework is employed to take into account of the undesirable effects of system disturbance (or noise) in control inputs such that a cost function achieves its minimum even in the worst disturbances of the system. For the purpose, we replace the bilinear multiplier $q$ in Equation (1) by $c+\eta$, where $c$ is a control variable that belongs to the admissible control set $\mathscr{C}_{\text {ad }}$, and $\eta$ is a disturbance (or noise) that belongs to the admissible disturbance set $\mathscr{D}_{\text {ad }}$. We also introduce the following cost function to be minimized within $\mathscr{C}_{\text {ad }}$ and maximized within $\mathscr{D}_{\text {ad }}$ :

$$
J(c, \eta)=\frac{1}{2}\left\|u-z_{d}\right\|_{L^{2}(Q)}^{2}+\frac{\alpha}{2}\|c\|_{L^{2}(Q)}^{2}-\frac{\beta}{2}\|\eta\|_{L^{2}(Q)}^{2},
$$

where $u$ is a solution of Equation (1), $z_{d} \in \mathrm{L}^{2}(Q)$ is desired value, and the positive constants $\alpha$ and $\beta$ are the relative weights of the second and third terms on the right hand side of (3).

Our goal of this paper is to find and characterize the optimal control of the cost function (3) for the worst disturbance through control input in Equation (1).

This leads to the problem of finding and characterizing the saddle point $\left(c^{*}, \eta^{*}\right) \in \mathscr{C}_{\mathrm{ad}} \times \mathscr{D}_{\mathrm{ad}}$ satisfying

$$
J(c *, \eta) \leq J(c *, \eta *) \leq J(c, \eta *), \quad \forall(c, \eta) \in \mathscr{C}_{\mathrm{ad}} \times \mathscr{D}_{\mathrm{ad}}
$$

In this paper, we use the terminology optimal pair for such a saddle point $\left(c^{*}, \eta^{*}\right)$ in (4). For the study of the existence of an optimal pair $\left(c^{*}, \eta^{*}\right)$ satisfying (4), we can find results in [8]. In that paper, the author used the minimax theorem in infinite dimensions given in Barbu and Precupanu [9]. And in [10], we extended the result to a quasilinear PDE.

On the other hand, in this paper, we use the method given in [11] to obtain the uniqueness as well as the existence of an optimal pair. That is to say, we use the strict convexity (or concavity) arguments of [12] by proving twice (Fréchet) differentiability of the solution map. Also, as we will see later, this method can suggest another condition that ensure strict convexity (or concavity) of the map from control (or noise) to the quadratic cost function (3).

Next, we derive an optimality condition for such a $\left(c^{*}, \eta^{*}\right)$ in (4). To derive the condition, we refer to the studies on bilinear optimal control problems where the state equations are linear partial differential equations such as the reaction diffusion equation or Kirchhoff plate equation (see [1, 3, 8, 13] and references therein).

We now explain the content of this paper. In Section 2, we present notations and some necessary lemmas. In Section
3, we prove the well-posedness of Equation (1) with respect to $u$ in the Hadamard sense using some previous results. To name just a few, we can refer to [14-16], and references therein. Especially, in order to prove the local Lipschitz continuity of the nonlinear solution map, we employ the energy equality of Volterra-type integro-differential equation which is proved in [17]. In Section 4, we shall study the minimax optimal control problems: at first, we shall show that the solution map of Equation (1): $q \longrightarrow u$ is the first and twice Fréchet differentiable; By using twice Fréchet differentiability of the solution maps $c \longrightarrow u$ and $\eta \longrightarrow u$, we prove that the maps $c \longrightarrow J$ and $\eta \longrightarrow J$ are strictly convex and concave, respectively, under the assumptions that $\alpha, \beta$ are sufficiently large or $T>0$ is sufficiently small. And we also prove that the maps $c \longrightarrow J$ and $\eta \longrightarrow J$ are lower and upper semicontinuous, respectively. Consequently, we can prove the uniqueness and existence of an optimal pair. Next, we derive the necessary optimality condition of an optimal pair for the observation case associated with the cost (3).

\section{Notations and Preliminaries}

Throughout this paper, we use $C$ as a generic constant and omit the integral variables in any definite integrals without confusion.

If $X$ is a Banach space, we denote by $X^{\prime}$ its topological dual, and by $\langle\cdot, \cdot\rangle_{X^{\prime}, X}$ the duality pairing between $X^{\prime}$ and $X$. We introduce the following abbreviations:

$$
L^{p}=L^{p}(\Omega), \quad W^{k, p}=W^{k, p}(\Omega), \quad\|\cdot\|_{p}=\|\cdot\|_{L^{p}},
$$

where $p \geq 1$ and $W^{k, p}$ is the $L^{p}$-based Sobolev spaces for $k \geq 1$. We denote by $H^{k}$, the standard Sobolev spaces $W^{k, 2}$ for $k \geq 1$. And $H_{0}^{k}$ means the completions of $C_{0}^{\infty}(\Omega)$ in $H^{k}$ for $k \geq 1$. The duality pairs between $H_{0}^{k}$ and $H^{-k}(k=1,2)$ are abbreviated by $\langle\cdot, \cdot\rangle_{k,-k}$. The scalar product and norm on $L^{2}$ are denoted by $(\cdot, \cdot)_{2}$ and $\|\cdot\|_{2}$, respectively. Then, based on the Poincaré inequality and the well-known regularity theory for elliptic boundary value problems (Temam [18] p. 150), the scalar products on $H_{0}^{k}(k=1,2)$ can be given as follows:

$$
\begin{aligned}
& ((v, o))_{H_{0}^{1}}=(\nabla v, \nabla o)_{2}, \forall v, o \in H_{0}^{1} \\
& ((v, o))_{H_{0}^{2}}=(\Delta v, \Delta o)_{2}, \forall v, o \in H_{0}^{2}
\end{aligned}
$$

Then obviously,

$$
\|v\|_{H_{0}^{1}}=\|\nabla v\|_{2}, \forall v \in H_{0}^{1}, \quad\|o\|_{H_{0}^{2}}=\|\Delta o\|_{2}, \quad \forall o \in H_{0}^{2} .
$$

We define the operator $A$ which stands for the following:

$$
\mathrm{Au}=-\Delta u, \quad V \equiv D(A)=H^{2} \cap H_{0}^{1},
$$


and consider the operator $M_{\gamma}=I+\gamma A$. We also define the operator $\mathscr{A}$ as follows:

$$
\mathcal{A u}=\Delta^{2} u, \quad D(\mathscr{A})=\left\{u \in H^{4} \cap H_{0}^{1} \mid \Delta u=0 \text { on } \Gamma\right\} .
$$

We note that

$$
D\left(\mathscr{A}^{1 / 2}\right)=V
$$

By using again the well-known elliptic regularity theory (Temam [18] p. 150), we can obtain

$\|v\|_{H^{2}} \leq C\|\Delta v\|_{2}, \quad \forall v \in V, \quad\|o\|_{H^{4}} \leq C\left\|\Delta^{2} o\right\|_{2}, \quad \forall o \in D(\mathscr{A})$

Therefore, we can employ

$\|v\|_{V}=\|\Delta v\|_{2}, \quad \forall v \in V, \quad\|o\|_{D(\mathscr{A})}=\left\|\Delta^{2} o\right\|_{2}, \quad \forall o \in D(\mathscr{A})$.

It becomes apparent that each topological imbedding

$$
V \longrightarrow H_{0}^{1} \longrightarrow L^{2} \longrightarrow H^{-1} \longrightarrow V^{\prime}
$$

is continuous and compact. According to Adams [19], we know that when $n \leq 3$, the imbedding

$$
V \longrightarrow C^{0}(\bar{\Omega})
$$

is compact.

It is well known that the biharmonic operator

$$
\Delta^{2}: H^{4} \cap H_{0}^{2} \longrightarrow L^{2}
$$

is bijective, and it admits an isometric extension

$$
\Delta^{2}: H_{0}^{2} \longrightarrow H^{-2}
$$

Thus, we can define an operator $G \in \mathscr{L}\left(L^{2}, H^{4} \cap H_{0}^{2}\right)$ (or $\left.\mathscr{L}\left(H^{-2}, H_{0}^{2}\right)\right)$ by

$$
G f=g \text { iff } \Delta^{2} g=f \text { in } \Omega, \quad g=\frac{\partial g}{\partial v}=0 \text { on } \quad \partial \Omega .
$$

Therefore, from Equation (1), one can also note that

$$
v=-G[u, u] \quad \forall u \in H_{0}^{2} .
$$

We collect below some results for the Airy stress function and von Kárman bracket.

Lemma 1. The trilinear form $b: V \times V \times V \longrightarrow R$ given by

$$
b(v, o, \varphi) \equiv([v, o], \varphi)_{2}
$$

satisfies the property

$$
b(v, o, \varphi)=b(v, \varphi, o), \quad \forall v, o, \varphi \in V
$$

Proof. See ([20], Proposition 1.4.2).

Lemma 2. The bilinear forms $(v, o) \longrightarrow G[v, o]$ from $H^{2} \times H^{2}$ into $W^{2, \infty}$ and $(v, o) \longrightarrow[v, o]$ from $H^{1} \times H^{2}$ into $H^{-2}$ are continuous. We also have the following estimates:

$$
\begin{aligned}
&\|G[v, o]\|_{W^{2, \infty}} \leq C\|v\|_{H^{2}}\|o\|_{H^{2}}, \quad \forall v, o \in H^{2}, \\
&\|[v, o]\|_{H^{-2}} \leq C\|v\|_{H^{1}}\|o\|_{H^{2}}, \quad \forall v \in H^{1}, \quad \forall o \in H^{2} .
\end{aligned}
$$

\section{Consequently,}

$$
\|[\varphi, G[v, o]]\|_{2} \leq C\|\varphi\|_{H^{2}}\|v\|_{H^{2}}\|o\|_{H^{2}}, \quad \forall \varphi, v, o \in H^{2} .
$$

Proof. See $[15,20]$.

\section{Well Posedness of a von Kárman Equation with Long Memory}

We introduce the Hilbert space $\mathcal{W}(0, T)$ of the weak solutions of Equation (1) given by

$$
\mathcal{W}(0, T)=\left\{u \mid u \in L^{2}(0, T ; V), u^{\prime} \in L^{2}\left(0, T ; H_{0}^{1}\right), u^{\prime \prime} \in L^{2}(Q)\right\}
$$

with the norm

$$
\|u\|_{w(0, T)}=\left(\|u\|_{L^{2}(0, T ; V)}^{2}+\left\|u^{\prime}\right\|_{L^{2}\left(0, T ; H_{0}^{1}\right)}^{2}+\left\|u^{\prime \prime}\right\|_{L^{2}(Q)}^{2}\right)^{1 / 2} .
$$

Definition 3. Function $u \in \mathcal{W}(0, T)$ is called a weak solution of Equation (1), if it satisfies

$$
\left\{\begin{array}{l}
\left\langle M_{\gamma} u^{\prime \prime}(\cdot), o\right\rangle_{V^{\prime}, V}+(\Delta u(\cdot)+k * \Delta u(\cdot), \Delta o)_{2}=([u(\cdot), v(\cdot)]+q(\cdot) u(\cdot)+f(\cdot), o)_{2}, \\
(\Delta v(\cdot), \Delta \varphi)_{2}=-([u(\cdot), u(\cdot)], \varphi)_{2}, \\
\text { for all }(o, \varphi) \in V \times H_{0}^{2} \text { in the sense of } \mathscr{D}^{\prime}(0, T), \\
u(0)=u_{0}, u^{\prime}(0)=u_{1},
\end{array}\right.
$$


where $\mathscr{D}^{\prime}(0, T)$ is the space of distributions on $(0, T)$.

As indicated in [14], von Kárman nonlinearity is subcritical; thus, the issues of well-posedness and regularity of weak solutions are standard.

Theorem 4. If $\left(u_{0}, u_{1}\right) \in V \times H_{0}^{1}, k \in C^{1}([0, T]), q \in L^{\infty}(Q)$, and $f \in L^{2}(Q)$, then a weak solution $u$ of Equation (1) exists and satisfies:

$$
u \in \mathcal{W}(0, T) \cap L^{\infty}(0, T ; V) \cap W^{1, \infty}\left(0, T ; H_{0}^{1}\right) .
$$

To show the regularity of a weak solutions of Equation (1), we need the following lemma.

Lemma 5. Let $X, Y$ be two Banach spaces, $X \subset Y$ with dense, and $X$ being reflexive. Set

$C_{w}([0, T] ; Y)=\left\{f \in L^{\infty}(0, T ; Y) \mid\langle f(\cdot), \xi\rangle_{Y, Y^{\prime}} \in C([0, T]), \quad \forall \xi \in Y^{\prime}\right\}$.

Then

$$
L^{\infty}(0, T ; X) \cap C_{w}([0, T] ; Y)=C_{w}([0, T] ; X) .
$$

Proof. See ([21], p. 275).

Corollary 6. Assume that $u$ is a weak solution of Equation (1). Then, we can assert (after possibly a modification on a set of measure zero) that

$$
u \in C_{w}([0, T] ; V), \quad u^{\prime} \in C_{w}\left([0, T] ; H_{0}^{1}\right) .
$$

Proof. From Dautray and Lions ([22], p. 480), it is clear that $\mathcal{W}(0, T) \hookrightarrow C\left([0, T] ; H_{0}^{1}\right) \cap C^{1}\left([0, T] ; L^{2}\right)$. Therefore, since $u$ $\in \mathcal{W}(0, T) \cap L^{\infty}(0, T ; V) \cap W^{1, \infty}\left(0, T ; H_{0}^{1}\right)$, the proof is the immediate consequence of Lemma 5 obtained by setting $X=V, Y=H_{0}^{1}$ to have $u \in C_{w}([0, T] ; V)$ and by setting $X=$ $H_{0}^{1}, Y=L^{2}$ to have $u^{\prime} \in C_{w}\left([0, T] ; H_{0}^{1}\right)$.

In the sequel, we give the important energy equality of weak solutions of Equation (1). It is used to prove the improved regularity of weak solutions of Equation (1) and used in all estimations in this paper.

Lemma 7. Assume that $u$ is a weak solution of Equation (1). Then, for each $t \in[0, T]$, we have the energy equality

$$
\begin{aligned}
& \left\|u^{\prime}(t)\right\|_{2}^{2}+\gamma\left\|\nabla u^{\prime}(t)\right\|_{2}^{2}+\|\Delta u(t)\|_{2}^{2} \\
& \quad+\frac{1}{2}\|\Delta v(t)\|_{2}^{2}+2(k * \Delta u(t), \Delta u(t))_{2} \\
& =2 \int_{0}^{t}\left(k^{\prime} * \Delta u, \Delta u\right)_{2} d s+2 \int_{0}^{t} k(0)\|\Delta u\|_{2}^{2} d s+2 \int_{0}^{t}\left(q u, u^{\prime}\right)_{2} d s \\
& \quad+2 \int_{0}^{t}\left(f, u^{\prime}\right)_{2} d s+\left\|u_{1}\right\|_{2}^{2}+\gamma\left\|\nabla u_{1}\right\|_{2}^{2}+\left\|\Delta u_{0}\right\|_{2}^{2}+\frac{1}{2}\left\|\Delta v_{0}\right\|_{2}^{2},
\end{aligned}
$$

where $\Delta v_{0}=-\Delta^{-1}\left[u_{0}, u_{0}\right]$.
Proof. By Corollary 6 and the uniform boundedness theorem, we have $u(t) \in V$ and $u^{\prime}(t) \in H_{0}^{1}$ for all $t \in[0, T]$. Thus, every function in (30) has meaning for all $t \in[0, T]$. Then, we can proceed the proof as in ([17], Proposition 2.1). By regarding $f$ in ([17], Proposition 2.1) as $[u, v]+q u+f$ in Equation (1), we can deduce that the weak solution $u$ of Equation (1) satisfies

$$
\begin{aligned}
& \left\|u^{\prime}(t)\right\|_{2}^{2}+\gamma\left\|\nabla u^{\prime}(t)\right\|_{2}^{2}+\|\Delta u(t)\|_{2}^{2}+2(k * \Delta u(t), \Delta u(t))_{2} \\
& =2 \int_{0}^{t}\left(k^{\prime} * \Delta u, \Delta u\right)_{2} \mathrm{ds}+2 \int_{0}^{t} k(0)\|\Delta u\|_{2}^{2} \mathrm{ds} \\
& \quad+2 \int_{0}^{t}\left([u, v]+\mathrm{qu}+f, u^{\prime}\right)_{2} \mathrm{~d} s+\left\|u_{1}\right\|_{2}^{2}+\gamma\left\|\nabla u_{1}\right\|_{2}^{2}+\left\|\Delta u_{0}\right\|_{2}^{2} .
\end{aligned}
$$

From [4], we can have

$$
2 \int_{0}^{t}\left([u, v], u^{\prime}\right)_{2} \mathrm{ds}=-\frac{1}{2}\|\Delta v(t)\|_{2}^{2}+\frac{1}{2}\left\|\Delta v_{0}\right\|_{2}^{2}
$$

Thus, we have (30).

This proves the lemma.

From the energy equalities (30) or (31) together with the following well-known Gronwall's lemma, we can prove uniqueness and regularity of weak solutions of Equation (1).

Lemma 8. Let $\xi(\cdot)$ be a nonnegative, absolutely continuous function on $[0, T]$, which satisfies the differentiable inequality for a.e.t $\in[0, T]$ :

$$
\xi^{\prime}(t) \leq \psi(t) \xi(t)+\phi(t)
$$

where $\psi$ and $\phi$ are nonnegative, summable functions on $[0, T]$. Then

$$
\xi(t) \leq e \int_{0}^{t} \psi d s\left(\xi(0)+\int_{0}^{t} \phi d s\right)
$$

for all $0 \leq t \leq T$.

Proof. See ([23], p.624).

Here, we can state the following theorem.

Theorem 9. Assume that $\left(u_{0}, u_{1}\right) \in V \times H_{0}^{1}, k \in C^{1}([0, T])$, $q \in L^{\infty}(Q)$, and $f \in L^{2}(Q)$. Then Equation (1) has a unique weak solution $u$ in $\mathcal{S}(0, T) \equiv \mathcal{W}(0, T) \cap C([0, T] ; V) \cap C^{l}$ $\left([0, T] ; H_{0}^{1}\right)$. Moreover, the solution mapping $p=\left(u_{0}, u_{1}, q\right.$, $f) \longrightarrow\left(u(p), u^{\prime}(p), v(p)\right)$ of $\mathscr{P} \equiv V \times H_{0}^{1} \times L^{\infty}(Q) \times L^{2}(Q)$ into $C([0, T] ; V) \times C\left([0, T] ; H_{0}^{1}\right) \times C\left([0, T] ; W^{2, \infty}\right)$ is locally Lipschitz continuous. 
Indeed, let $p_{1}=\left(u_{0}^{1}, u_{1}^{1}, q_{1}, f_{1}\right) \in \mathscr{P}$ and $p_{2}=\left(u_{0}^{2}, u_{1}^{2}, q_{2}\right.$, $\left.f_{2}\right) \in \mathscr{P}$, we prove this theorem by showing the following inequality

$$
\begin{aligned}
& \left\|u\left(p_{1}\right)-u\left(p_{2}\right)\right\|_{\mathcal{S}(0, T)}+\left\|v\left(p_{1}\right)-v\left(p_{2}\right)\right\|_{C\left([0, T] ; W^{2, \infty}\right)} \\
& \quad \leq C\left\|p_{1}-p_{2}\right\|_{\mathscr{P}}
\end{aligned}
$$

where $C>0$ is a constant depending on the data and

$$
\begin{aligned}
& \left\|p_{1}-p_{2}\right\|_{\mathscr{P}} \\
& \quad=\left(\left\|u_{0}^{1}-u_{0}^{2}\right\|_{V}^{2}+\left\|u_{1}^{1}-u_{1}^{2}\right\|_{H_{0}^{1}}^{2}+\left\|q_{1}-q_{2}\right\|_{L^{\infty}(Q)}^{2}+\left\|f_{1}-f_{2}\right\|_{L^{2}(Q)}^{2}\right)^{1 / 2} .
\end{aligned}
$$

Proof of Theorem 9. Lemma 7 allows us to show the regularity of $u$. It is verified from the data conditions that the right hand side of (30) is continuous in $t$. Hence, we have that

$$
t \longrightarrow\left\|\nabla u^{\prime}(t)\right\|_{2}^{2}+\|\Delta u(t)\|_{2}^{2}
$$

is continuous on $[0, T]$. Indeed, $u \in C([0, T] ; V) \cap C^{1}([0$, $\left.T] ; H_{0}^{1}\right)$.

Therefore, considering results in $[15,16]$ and $[14]$, we can deduce that Equation (1) possesses a unique weak solution $u \in \mathcal{S}(0, T)$ under the data condition $\left(u_{0}, u_{1}, f\right) \in \mathcal{H} \equiv V \times$ $H_{0}^{1} \times L^{2}(Q)$ such that

$$
\|u\|_{\mathcal{S}(0, T)} \leq C\left\|\left(u_{0}, u_{1}, f\right)\right\|_{\mathscr{H}} .
$$

Based on the above result, we prove the inequality (35). For the purpose, we denote $u_{1}-u_{2} \equiv u\left(p_{1}\right)-u\left(p_{2}\right)$ by $\phi$ and $v_{1}-v_{2} \equiv v\left(p_{1}\right)-v\left(p_{2}\right)$ by $Y$. Then, we can know from Equation (1) that $\phi$ and $Y$ satisfy the following equation in the weak sense:

$$
\left\{\begin{array}{l}
M_{\gamma} \phi^{\prime \prime}+\Delta^{2} \phi+k * \Delta^{2} \phi=\left[\phi, v_{1}\right]+\left[u_{2}, Y\right]+q_{1} \phi+\left(q_{1}-q_{2}\right) u_{2}+f_{1}-f_{2}, \\
\Delta^{2} Y=-\left[\phi, u_{1}+u_{2}\right] \text { in } Q, \\
\phi=\Delta \phi=Y=\frac{\partial Y}{\partial \nu}=0 \text { on } \Sigma, \\
\phi(0)=u_{0}^{1}-u_{0}^{2}, \phi^{\prime}(0)=u_{1}^{1}-u_{1}^{2} \text { in } \Omega .
\end{array}\right.
$$

We note that

$$
\left[u_{2}, Y\right]=\left[u_{2},-G\left[\phi, u_{1}+u_{2}\right]\right]
$$

Just as deriving the equality (31) from Equation (1), we can know that the weak solution $\phi$ of Equation (39) satisfies

$$
\begin{aligned}
\left\|\phi^{\prime}(t)\right\|_{2}^{2}+\gamma\left\|\nabla \phi^{\prime}(t)\right\|_{2}^{2}+\|\Delta \phi(t)\|_{2}^{2} \\
=-2(k * \Delta \phi(t), \Delta \phi(t))_{2}+2 \int_{0}^{t}\left(k^{\prime} * \Delta \phi, \Delta \phi\right)_{2} \mathrm{ds} \\
\quad+2 \int_{0}^{t} k(0)\|\Delta \phi\|_{2}^{2} \mathrm{~d} s+2 \int_{0}^{t}\left(\left[\phi, v_{1}\right]+\left[u_{2}, \mathrm{Y}\right]+q_{1} \phi\right. \\
\left.\quad+\left(q_{1}-q_{2}\right) u_{2}, \phi^{\prime}\right)_{2} \mathrm{ds}+2 \int_{0}^{t}\left(f_{1}-f_{2}, \phi^{\prime}\right)_{2} \mathrm{ds} \\
\quad+\left\|\phi^{\prime}(0)\right\|_{2}^{2}+\gamma\left\|\nabla \phi^{\prime}(0)\right\|_{2}^{2}+\|\Delta \phi(0)\|_{2}^{2} .
\end{aligned}
$$

At first, we note by (13) that:

$$
\begin{aligned}
&\left|2 \int_{0}^{t}\left(q_{1} \phi, \phi^{\prime}\right)_{2} \mathrm{ds}\right| \leq 2\left\|q_{1}\right\|_{L^{\infty}(Q)} \int_{0}^{t}\|\phi\|_{2}\left\|\phi^{\prime}\right\|_{2} \mathrm{ds} \\
& \leq C \int_{0}^{t}\|\Delta \phi\|_{2}\left\|\phi^{\prime}\right\|_{2} \mathrm{ds} \\
& \leq C \int_{0}^{t}\left(\|\Delta \phi\|_{2}^{2}+\left\|\phi^{\prime}\right\|_{2}^{2}\right) \mathrm{ds}, \\
&\left|2 \int_{0}^{t}\left(\left(q_{1}-q_{2}\right) u_{2}, \phi^{\prime}\right){ }_{2} \mathrm{ds}\right| \\
& \leq 2 \int_{0}^{t}\left\|\left(q_{1}-q_{2}\right) u_{2}\right\|_{2}\left\|\phi^{\prime}\right\|_{2} \mathrm{ds} \\
& \leq\left\|\left(q_{1}-q_{2}\right) u_{2}\right\|_{L^{2}(Q)}^{2}+\int_{0}^{t}\left\|\phi^{\prime}\right\|_{2}^{2} \mathrm{ds} \\
& \leq\left\|q_{1}-q_{2}\right\|_{L^{\infty}(Q)}^{2}\left\|u_{2}\right\|_{L^{2}(Q)}^{2}+\int_{0}^{t}\left\|\phi^{\prime}\right\|_{2}^{2} \mathrm{ds} .
\end{aligned}
$$

By $u_{2} \in \mathcal{S}(0, T) \hookrightarrow L^{2}(Q)$ and (38), we can get from (43) that

$$
\left|2 \int_{0}^{t}\left(\left(q_{1}-q_{2}\right) u_{2}, \phi^{\prime}\right)_{2} \mathrm{ds}\right| \leq C\left\|q_{1}-q_{2}\right\|_{L^{\infty}(Q)}^{2}+\int_{0}^{t}\left\|\phi^{\prime}\right\|_{2}^{2} \mathrm{ds} .
$$

For other estimates of the remaining terms on the right hand side of (41), we can refer to the previous results in [4] and obtain with (41) and (42)-(44) the following:

$$
\begin{aligned}
& \left\|\phi^{\prime}(t)\right\|_{2}^{2}+\left\|\nabla \phi^{\prime}(t)\right\|_{2}^{2}+\|\Delta \phi(t)\|_{2}^{2} \\
& \leq C\left(\left\|\phi^{\prime}(0)\right\|_{2}^{2}+\left\|\nabla \phi^{\prime}(0)\right\|_{2}^{2}+\|\Delta \phi(0)\|_{2}^{2}+\| q_{1}\right. \\
& \left.\quad-q_{2}\left\|_{L^{\infty}(Q)}^{2}+\right\| f_{1}-f_{2} \|_{L^{2}(Q)}^{2}+\int_{0}^{t}\left(\|\Delta \phi\|_{2}^{2}+\left\|\phi^{\prime}\right\|_{2}^{2}\right) \mathrm{ds}\right) \\
& \leq C\left(\left\|p_{1}-p_{2}\right\|_{\mathscr{P}}^{2}+\int_{0}^{t}\left(\|\Delta \phi\|_{2}^{2}+\left\|\phi^{\prime}\right\|_{2}^{2}\right) \mathrm{ds}\right) .
\end{aligned}
$$


By applying Lemma 8 to (45), we have

$$
\left\|\nabla \phi^{\prime}(t)\right\|_{2}^{2}+\|\Delta \phi(t)\|_{2}^{2} \leq C\left\|p_{1}-p_{2}\right\|_{\mathscr{P}}^{2}
$$

And also, for almost $t \in[0, T]$, we obtain by Lemma 2 and (11) that

$$
\begin{aligned}
\|Y(t)\|_{W^{2, \infty}}^{2} & =\left\|-G\left[\phi(t), u_{1}(t)+u_{2}(t)\right]\right\|_{W^{2, \infty}}^{2} \\
& \leq C\|\Delta \phi(t)\|_{2}^{2}\left\|\Delta\left(u_{1}(t)+u_{2}(t)\right)\right\|_{2}^{2} \\
& \leq C\left(\left\|u_{1}\right\|_{\mathcal{S}(0, T)}^{2}+\left\|u_{2}\right\|_{\mathcal{S}(0, T)}^{2}\right)\|\Delta \phi(t)\|_{2}^{2} .
\end{aligned}
$$

By (38), (46), and (47), we can obtain

$$
\|Y(t)\|_{W^{2, \infty}}^{2} \leq C\left\|p_{1}-p_{2}\right\|_{\mathscr{P}}^{2} .
$$

This implies with (46) that

$$
\|\phi\|_{C([0, T] ; V) \cap C^{1}\left([0, T] ; H_{0}^{1}\right)}+\|Y\|_{C\left([0, T] ; W^{2, \infty}\right)} \leq C\left\|p_{1}-p_{2}\right\|_{\mathscr{P}} .
$$

Since $M_{\gamma}^{-1} A^{1 / 2} \in \mathcal{L}\left(L^{2}\right)$ and $M_{\gamma}^{-1} \in \mathcal{L}\left(L^{2}, V\right)$, by conducting similar estimations in Equation (39), we can obtain from (49) that

$$
\left\|\phi^{\prime \prime}\right\|_{L^{2}(Q)} \leq C\left\|p_{1}-p_{2}\right\|_{P}
$$

Hence, by (49) and (50) we can prove (35). This completes the proof.

\section{Quadratic Cost Minimax Control Problems}

Let the following be the set of the admissible controls:

$$
\mathcal{C}_{\mathrm{ad}}=\left\{c \in L^{\infty}(Q) \mid \underline{c} \leq c \leq \bar{c} \text { a.e.in } Q\right\},
$$

where $\underline{c}$ and $\bar{c}$ are given constants, representing lower and upper bounds of the admissible control variables, respectively. Let the following be the set of the admissible disturbances or noises:

$$
\mathcal{D}_{\mathrm{ad}}=\left\{\eta \in L^{\infty}(Q) \mid \underline{\eta} \leq \eta \leq \bar{\eta} \text { a.e.in } Q\right\},
$$

where $\eta$ and $\bar{\eta}$ are given constants, representing lower and upper bounds of admissible disturbance variables, respectively. For variational analysis, we use the $L^{2}(Q)$ norm on $\mathcal{C}_{\text {ad }}$ and $\mathcal{D}_{\text {ad }}$. For simplicity, we denote by $\mathcal{B}_{\text {ad }}=\mathcal{C}_{\text {ad }} \times \mathcal{D}_{\text {ad }}$. From Theorem 9, we can uniquely define the solution map $\mathcal{B}_{\mathrm{ad}} \longrightarrow \mathcal{S}(0, T)$, which maps from $q=(c, \eta) \in \mathcal{B}_{\mathrm{ad}}$ to the weak solution $u(q) \in \mathcal{S}(0, T)$, where $u(q)$ satisfies the following equation:

$$
\left\{\begin{array}{l}
M_{\gamma} u^{\prime \prime}(q)+\Delta^{2} u(q)+\int_{0}^{t} k(t-s) \Delta^{2} u(q ; s) \mathrm{d} s=[u(q), v(q)]+(c+\eta) u(q)+f \\
\Delta^{2} v(q)=-[u(q), u(q)] \text { in } Q \\
u(q)=\Delta u(q)=v(q)=\frac{\partial v(q)}{\partial v}=0 \text { on } \Sigma \\
u(q ; 0, x)=u_{0}(x), u^{\prime}(q ; 0, x)=u_{1}(x) \text { in } \Omega
\end{array}\right.
$$

The weak solution $u(q)$ of Equation (53) is called the state of the control system Equation (53).

To study the quadratic cost minimax optimal control problems for Equation (53), we introduce the following quadratic cost function

$$
J(c, \eta)=\frac{1}{2}\left\|u(q)-z_{d}\right\|_{L^{2}(Q)}^{2}+\frac{\alpha}{2}\|c\|_{L^{2}(Q)}^{2}-\frac{\beta}{2}\|\eta\|_{L^{2}(Q)}^{2}
$$

where $z_{d} \in L^{2}(Q)$ is the desired value, and the positive constants $\alpha$ and $\beta$ are the relative weights of the second and third terms on the right hand side of (54).

As indicated in the introduction, we shall study the minimax optimal control problem as follows: we prove the uniqueness as well as existence of a control $c^{*} \in \mathcal{C}_{\text {ad }}$ and a dis- turbance (or noise) $\eta^{*} \in \mathcal{D}_{\text {ad }}$ such that $\left(c^{*}, \eta^{*}\right)$ is a saddle point of the functional $J(\cdot, \cdot)$ of $(54)$. That is,

$$
J\left(c^{*}, \eta\right) \leq J\left(c^{*}, \eta^{*}\right) \leq J\left(c, \eta^{*}\right), \forall(c, \eta) \in \mathcal{B}_{\mathrm{ad}} .
$$

Here, we call such $\left(c^{*}, \eta^{*}\right)$ in (55) to be an optimal pair for the minimax optimal control problem with the cost (54). And we need to characterize $\left(c^{*}, \eta^{*}\right)$ in (55) by giving the necessary optimality condition through adjoint equation related to Equation (53) and the cost (54). For this purpose, we have to show the differentiabilities of the control to state mapping.

4.1. Differentiabilities of the Nonlinear Solution Map. We study the Fréchet differentiability of the nonlinear solution map, which is an improvement of the previous results in 
[4] and is more desirable for many applications. From Theorem 9, for fixed $\left(u_{0}, u_{1}, f\right) \in V \times H_{0}^{1} \times L^{2}(Q)$ in Equation (53), we know that the solution map $L^{\infty}(Q) \longrightarrow \mathcal{S}(0, T)$ from $q\left(=c+\eta\right.$ in Equation (53)) $\in L^{\infty}(Q)$ to $u(q) \in \mathcal{S}(0, T)$ is well defined and continuous.

For our study, we present the following definitions.

Definition 10. The solution map $q \longrightarrow u(q)$ of $L^{\infty}(Q)$ into $\mathcal{S}(0, T)$ is said to be Fréchet differentiable on $L^{\infty}(Q)$ if for any $q \in L^{\infty}(Q)$, there exists a $T(q) \in \mathscr{L}\left(L^{\infty}(Q), \mathcal{S}(0, T)\right)$ such that, for any $w \in L^{\infty}(Q)$,

$$
\lim _{\|w\|_{L^{\infty}(Q)} \longrightarrow 0} \frac{\|u(q+w)-u(q)-T(q) w\|_{\mathcal{S}(0, T)}}{\|w\|_{L^{\infty}(Q)}}=0 .
$$

The operator $T(q)$ is called the Fréchet derivative of $u$ at $q$, which we denote by $D u(q) . T(q) w=D u(q) w \in \mathcal{S}(0, T)$ is called the Fréchet derivative of $u$ at $q$ in the direction of $w \in L^{\infty}(Q)$.

Definition 11. Let $U$ be a subset of $L^{\infty}(Q)$ and $q, q^{*} \in \mathcal{U}$. The solution map $q \longrightarrow u(q)$ of $\mathcal{U}$ into $\mathcal{S}(0, T)$ is said to be Gâteaux differentiable at $q^{*}$ in the direction $q-q^{*}$ if there exists a function $\operatorname{Du}\left(q^{*} ; q-q^{*}\right) \in \mathcal{S}(0, T)$ such that

$$
\lim _{\lambda \longrightarrow 0}\left\|\frac{u\left(q^{*}+\lambda\left(q-q^{*}\right)\right)-u\left(q^{*}\right)}{\lambda}-\operatorname{Du}\left(q^{*} ; q-q^{*}\right)\right\|_{\mathcal{S}(0, T)}=0
$$

Theorem 12. The solution map $q \longrightarrow u(q)$ of $L^{\infty}(Q)$ into $\mathcal{S}$ $(0, T)$ is Fréchet differentiable on $L^{\infty}(Q)$ and the Fréchet derivative of $u(q)$ at $q$ in the direction $w \in L^{\infty}(Q)$, that is to say $z=D u(q) w$, is the weak solution of

$$
\left\{\begin{array}{l}
M_{\gamma} z^{\prime \prime}+\Delta^{2} z+\int_{0}^{t} k(t-s) \Delta^{2} z \mathrm{ds}=[z,-G[u(q), u(q)]]+2[u(q),-G[z, u(q)]]+q z+w u(q) \text { in } Q \\
z=\Delta z=0 \text { on } \Sigma \\
z(0)=0, z^{\prime}(0)=0 \text { in } \Omega .
\end{array}\right.
$$

We prove this theorem by two steps.

(i) For any $w \in L^{\infty}(Q)$, Equation (58) admits a unique weak solution $z \in \mathcal{S}(0, T)$, namely, there exists an operator $T \in \mathscr{L}\left(L^{\infty}(Q), \mathcal{S}(0, T)\right)$ satisfying $T w=$ $z(=z(w))$

(ii) We show that $\|u(q+w)-u(q)-z\|_{\mathcal{S}(0, T)} \leq C$ $\|w\|_{L^{\infty}(Q)}^{2}$.

Proof. (i) Let

$$
\mathscr{G}(u(q), z):=[z,-G[u(q), u(q)]]+2[u(q),-G[z, u(q)]]
$$

Then, we can estimate the right hand side of (59) as follows. By (11) and Lemma 2 we have

$$
\|[z,-G[u(q), u(q)]]\|_{2} \leq C\|z\|_{H^{2}}\|u(q)\|_{H^{2}}^{2} \leq C\|u(q)\|_{\mathcal{S}(0, T)}^{2}\|\Delta z\|_{2} .
$$

This implies with (38) that

$$
\|[z,-G[u(q), u(q)]]\|_{2} \leq C\|\Delta z\|_{2} .
$$

Similarly, we have

$$
\|2[u(q),-G[z, u(q)]]\|_{2} \leq C\|\Delta z\|_{2} .
$$

Hence, by (61) and (62), we note that

$$
\mathscr{G}(u(q), \cdot) \in \mathscr{L}\left(V, L^{2}\right)
$$

Taking into account $w u(q) \in L^{2}(Q)$ and (63), we can employ the linear theory in [17] (cf. [22]) to verify that Equation (58) admits a unique weak solution $z \in \mathcal{S}(0, T)$. And also by using the energy equality to be satisfied by $z$ as in (31) and following similar estimations in Theorem 9, we can know by (38) that the weak solution $z(=z(w))$ of Equation (58) satisfies

$$
\begin{aligned}
\|z(w)\|_{\mathcal{S}(0, T)} & \leq C\|\operatorname{wu}(q)\|_{L^{2}(Q)} \leq C\|w\|_{L^{\infty}(Q)}\|u(q)\|_{L^{2}(Q)} \\
& \leq C\|u(q)\|_{\mathcal{S}(0, T)}\|w\|_{L^{\infty}(Q)} \leq C\|w\|_{L^{\infty}(Q)} .
\end{aligned}
$$

Hence, from (64), the mapping $w \in L^{\infty}(Q) \mapsto z(w) \in$ $\mathcal{S}(0, T)$ is linear and bounded. We can thus infer that there exists a $T \in \mathscr{L}\left(L^{\infty}(Q), \mathcal{S}(0, T)\right)$ such that $\mathrm{Tw}=z(w)$ for each $w \in L^{\infty}(Q)$. 
(ii) We set the difference $u(q+w)-u(q)-z=\theta$. Then, by noting the following:

$$
\begin{aligned}
& M_{\gamma} \theta^{\prime \prime}+\Delta^{2} \theta+k * \Delta^{2} \theta \\
&=[u(q+w), v(q+w)]-[u(q), v(q)]-[z, v(q)]-2[u(q), \\
&-G[z, u(q)]]+(q+w) u(q+w)-q u(q)-q z-w u(q) \\
&= {[u(q+w), v(q+w)-v(q)]+[\theta, v(q)] } \\
&-2[u(q),-G[z, u(q)]]+q \theta+w(u(q+w)-u(q)) \\
& \equiv {[u(q+w),-G[u(q+w), u(q+w)]} \\
&+G[u(q), u(q)]]+[\theta, v(q)]-2[u(q), \\
&-G[z, u(q)]]+q \theta+w(u(q+w)-u(q)) \\
&= {[u(q+w),-G[u(q+w)-u(q), u(q+w)+u(q)]] } \\
&+[\theta, v(q)]-2[u(q),-G[z, u(q)]]+(q+w) \theta+w z
\end{aligned}
$$

$$
\begin{aligned}
= & {[u(q+w),-G[\theta, u(q+w)+u(q)]] } \\
& +[u(q+w),-G[z, u(q+w)+u(q)]] \\
& +[\theta, v(q)]-2[u(q),-G[z, u(q)]]+(q+w) \theta+w z \\
= & {[u(q+w),-G[\theta, u(q+w)+u(q)]] } \\
& +[u(q+w)-u(q),-G[z, u(q+w)+u(q)]] \\
& +[u(q),-G[z, u(q+w)+u(q)]]+[\theta, v(q)] \\
& -2[u(q),-G[z, u(q)]]+(q+w) \theta+w z \\
= & {[u(q+w),-G[\theta, u(q+w)+u(q)]] } \\
& +[u(q+w)-u(q),-G[z, u(q+w)+u(q)]] \\
& +[u(q),-G[z, u(q+w)-u(q)]]+[\theta, v(q)] \\
& +(q+w) \theta+w z \text { in } Q,
\end{aligned}
$$

we know that $\theta$ satisfies

$$
\left\{\begin{array}{l}
M_{\gamma} \theta^{\prime \prime}+\Delta^{2} \theta+k * \Delta^{2} \theta=\Xi(u(q), u(q+w), \theta)+(q+w) \theta+w z+I_{1}+I_{2} \text { in } Q, \\
\theta=\Delta \theta=0 \text { on } \Sigma, \\
\theta(0, x)=0, \theta^{\prime}(0, x)=0 \text { in } \Omega,
\end{array}\right.
$$

in the weak sense, where

$$
\begin{aligned}
& \Xi(u(q), u(q+w), \theta) \\
& \quad=[u(q+w),-G[\theta, u(q+w)+u(q)]]+[\theta, v(q)], \\
& I_{1}=[u(q+w)-u(q),-G[z, u(q+w)+u(q)]], \\
& I_{2}=[u(q),-G[z, u(q+w)-u(q)]] .
\end{aligned}
$$

In a similar argument to (63), we know that

$$
\Xi(u(q), u(q+w), \cdot) \in \mathcal{L}\left(V, L^{2}\right)
$$

Thus with (68), we can apply the energy equality like (31) to (66) and follow similar estimations as in the Proof of Theorem 9 , to obtain

$$
\|\theta\|_{\mathcal{S}(0, T)} \leq C\left\|w z+I_{1}+I_{2}\right\|_{L^{2}(Q)} .
$$

By Theorem 9 and (64), we can deduce as follows.

$$
\begin{aligned}
\|w z\|_{L^{2}(Q)} & \leq\|w\|_{L^{\infty}(Q)}\|z\|_{L^{2}(Q)} \leq C\|w\|_{L^{\infty}(Q)}\|z\|_{\mathcal{S}(0, T)} \\
& \leq C\|w\|_{L^{\infty}(Q)}^{2} .
\end{aligned}
$$

By Lemma 2 and (11), we have

$$
\begin{aligned}
\left\|I_{1}\right\|_{L^{2}(Q)} \leq & C\|u(q+w)-u(q)\|_{C([0, T] ; V)}\|z\|_{C([0, T] ; V)} \\
& \times\|u(q+w)+u(q)\|_{L^{2}(0, T ; V)} \\
\leq & C\|u(q+w)-u(q)\|_{\mathcal{S}(0, T)}\|z\|_{\mathcal{S}(0, T)} \\
& \times\left(\|u(q+w)\|_{\mathcal{S}(0, T)}+\|u(q)\|_{\mathcal{S}(0, T)}\right) .
\end{aligned}
$$

From (38), (64) and Theorem 9, we can obtain with (71) that

$$
\left\|I_{1}\right\|_{L^{2}(Q)} \leq C\|w\|_{L^{\infty}(Q)}\|q+w-q\|_{L^{\infty}(Q)}=C\|w\|_{L^{\infty}(Q)}^{2} .
$$

By analogy with (71) and (72), we can deduce

$$
\left\|I_{2}\right\|_{L^{2}(Q)} \leq C\|w\|_{L^{\infty}(Q)}^{2}
$$

Hence, from (69) to (73), we can obtain

$$
\begin{aligned}
\|\theta\|_{\mathcal{S}(0, T)} & \leq C\left\|w z+I_{1}+I_{2}\right\|_{L^{2}(Q)} \\
& \leq C\left(\|w z\|_{L^{2}(Q)}+\left\|I_{1}\right\|_{L^{2}(Q)}+\left\|I_{2}\right\|_{L^{2}(Q)}\right) \\
& \leq C\|w\|_{L^{\infty}(Q)}^{2} .
\end{aligned}
$$

This completes the proof.

To show the uniqueness as well as existence of an optimal pair, we are going to use the strict convexity arguments in [12]. To this end, we consider the following results. 
Theorem 13. The map $q \longrightarrow u(q)$ of $L^{\infty}(Q)$ into $\mathcal{S}(0, T)$ is twice Fréchet differentiable at $q$ and the twice Fréchet deriva- tive of $u$ at $q$ in the direction $w \in L^{\infty}(Q)$, say $\rho=D^{2} u(q)(w$, $w)$, is a unique solution of the following problem

$$
\left\{\begin{array}{l}
M_{\gamma} \rho^{\prime \prime}+\Delta^{2} \rho+\int_{0}^{t} k(t-s) \Delta^{2} \rho d s=\mathcal{G}(u(q), \rho)+\mathscr{F}(z, u(q))+q \rho+2 w z \text { in } Q, \\
\rho=\Delta \rho=0 \text { on } \Sigma, \\
\rho(0)=0, \rho^{\prime}(0)=0 \text { in } \Omega,
\end{array}\right.
$$

where $z$ is the solution of Equation (58), $\mathscr{G}(u(q), \cdot)$ is given in (59), and

$$
\mathscr{F}(z, u(q))=4[z,-G[z, u(q)]]+2[u(q),-G[z, z]] .
$$

To prove Theorem 13, it is sufficient to show the following:

(i) $\|\rho\|_{\mathcal{S}(0, T)} \leq C\|w\|_{L^{\infty}(Q)}^{2}$;

(ii) $\|D u(q+w) w-D u(q) w-\rho\|_{\mathcal{S}(0, T)}=o\left(\|w\|_{L^{\infty}(Q)}\right)$ as $\|w\|_{L^{\infty}(Q)} \longrightarrow 0$.

Proof. (i) By (38) and (64), we can have the following estimate

$$
\begin{aligned}
\|\mathscr{F}(z, u(q))\|_{2} & \leq C\|u(q)\|_{H^{2}}\|z\|_{H^{2}}^{2} \leq C\|u(q)\|_{\mathcal{S}(0, T)}\|z\|_{\mathcal{S}(0, T)}^{2} \\
& \leq C\|w\|_{L^{\infty}(Q)}^{2} .
\end{aligned}
$$

Thus by similar arguments in the proof of (i) of Theorem 12 , we can show that the weak solution $\rho$ of Equation (75) can be estimated as follows:

$$
\|\rho\|_{\mathcal{S}(0, T)} \leq C\|\mathscr{F}(z, u(q))+2 w z\|_{L^{2}(Q)} .
$$

By (70) and (77), we know by (78) that

$$
\|\rho\|_{\mathcal{S}(0, T)} \leq C\|w\|_{L^{\infty}(Q)}^{2} .
$$

(ii) From Equation (58), we can deduce that $\kappa=\operatorname{Du}(q+$ $w) w$ is the weak solution of the following equation:

$$
\left\{\begin{array}{l}
M_{\gamma} \kappa^{\prime \prime}+\Delta^{2} \kappa+\int_{0}^{t} k(t-s) \Delta^{2} \kappa \mathrm{d} s=\mathscr{G}(u(q+w), \kappa)+(q+w) \kappa+w u(q+w) \text { in } Q, \\
\kappa=\Delta \kappa=0 \text { on } \Sigma, \\
\kappa(0)=0, \kappa^{\prime}(0)=0 \text { in } \Omega .
\end{array}\right.
$$

By previous result, we can verify the following

$$
\|\kappa\|_{\mathcal{S}(0, T)} \leq C\|w\|_{L^{\infty}(Q)} .
$$

From Equation (58), Equation (75) and Equation (80), $\chi=\kappa-z-\rho$ satisfies the following equation

$$
\left\{\begin{array}{l}
M_{\gamma} \chi^{\prime \prime}+\Delta^{2} \chi+\int_{0}^{t} k(t-s) \Delta^{2} \chi \mathrm{d} s=\mathscr{S}(u(q), \chi)+q \chi+\sum_{i=1}^{5} I_{i} \text { in } Q \\
\chi=\Delta \chi=0 \text { on } \Sigma \\
\chi(0)=0, \chi^{\prime}(0)=0 \text { in } \Omega,
\end{array}\right.
$$

in the weak sense, where

$$
\begin{aligned}
& I_{1}=[\kappa,-G[u(q+w)-u(q), u(q+w)+u(q)]], \\
& I_{2}=2[u(q+w)-u(q),-G[\kappa, u(q+w)]], \\
& I_{3}=2[u(q),-G[\kappa, u(q+w)-u(q)]], \\
& I_{4}=\mathscr{F}(z, u(q))(=4[z,-G[z, u(q)]]+2[u(q),-G[z, z]]), \\
& I_{5}=w \kappa+w \theta-w z,
\end{aligned}
$$


where $\theta=u(q+w)-u(q)-z$. By Theorem 9, (38) and (81), we can have

$$
\begin{aligned}
\left\|I_{1}\right\|_{2} \leq & C\|\kappa\|_{H^{2}}\|u(q+w)-u(q)\|_{H^{2}}\|u(q+w)+u(q)\|_{H^{2}} \\
\leq & C\left(\|u(q+w)\|_{\mathcal{S}(0, T)}+\|u(q)\|_{\mathcal{S}(0, T)}\right) \\
& \times\|\kappa\|_{\mathcal{S}(0, T)}\|u(q+w)-u(q)\|_{\mathcal{S}(0, T)} \\
\leq & C\|w\|_{L^{\infty}(Q)}^{2} .
\end{aligned}
$$

By analogy with (84), we obtain

$$
\left\|I_{2}\right\|_{2} \leq C\|w\|_{L^{\infty}(Q)}^{2} .
$$

Also, by analogy with (84), we have

$$
\left\|I_{3}\right\|_{2} \leq C\|w\|_{L^{\infty}(Q)}^{2}
$$

It holds by (77) that

$$
\left\|I_{4}\right\|_{2} \leq C\|w\|_{L^{\infty}(Q)}^{2} .
$$

By (81) and Theorem 12, we can have

$$
\begin{aligned}
\left\|I_{5}\right\|_{2} & \leq C\|w\|_{L^{\infty}(Q)}\left(\|\kappa\|_{\mathcal{S}(0, T)}+\|\theta\|_{\mathcal{S}(0, T)}+\|z\|_{\mathcal{S}(0, T)}\right) \\
& \leq C\left(\|w\|_{L^{\infty}(Q)}^{2}+\|w\|_{L^{\infty}(Q)}^{3}\right) .
\end{aligned}
$$

By similar arguments to those in the Proof of Theorem 9, we can deduce that the weak solution $\chi$ of Equation (82) satisfies

$$
\|\chi\|_{\mathcal{S}(0, T)} \leq C\left\|\sum_{i=1}^{5} I_{i}\right\|_{L^{2}(Q)} .
$$

From (84) to (89), we can deduce

$$
\|\chi\|_{\mathcal{S}(0, T)} \leq C\left(\|w\|_{L^{\infty}(Q)}^{3}+\|w\|_{L^{\infty}(Q)}^{2}\right),
$$

which implies

$$
\|\chi\|_{\mathcal{S}(0, T)}=o\left(\|w\|_{L^{\infty}(Q)}\right) \text { as }\|w\|_{L^{\infty}(Q)} \longrightarrow 0 .
$$

This completes the proof.

Corollary 14. The map $q \longrightarrow u(q)$ of $\mathcal{U} \subset L^{\infty}(Q)$ into $\mathcal{S}(0, T)$ is twice Gâteaux differentiable at $q^{*}$ and such the twice Gâteaux derivative of $u$ in the direction $q-q^{*}$, say $\rho=D^{2}$ $u\left(q^{*}\right)\left(q-q^{*}, q-q^{*}\right)$, is a unique solution of Equation (75) in which $q$ and $w$ are replaced by $q^{*}$ and $q-q^{*}$, respectively.

Proof. The proof is immediately followed by Theorem 13 .
Lemma 15. The weak solution $\rho$ of Equation (75) satisfies

$$
\|\rho\|_{\mathcal{S}(0, T)} \leq C\|w\|_{L^{2}(Q)}^{2} .
$$

Proof. Let $z(w)$ be the weak solution of Equation (58). Then, using $\mathcal{S}(0, T) \hookrightarrow C^{0}(\bar{Q})$, we can get from (64) the following:

$$
\begin{aligned}
\|z(w)\|_{\mathcal{S}(0, T)} & \leq C\|w u(q)\|_{L^{2}(Q)} \leq C\|u(q)\|_{C^{0}(\bar{Q})}\|w\|_{L^{2}(Q)} \\
& \leq C\|u(q)\|_{\mathcal{S}(0, T)}\|w\|_{L^{2}(Q)} \leq C\|w\|_{L^{2}(Q)} .
\end{aligned}
$$

Let $\rho$ be the weak solution of Equation (75). From the second inequality in (77) and (78), we can deduce with (38) and (93) the following:

$$
\begin{aligned}
\|\rho\|_{\mathcal{S}(0, T)} & \leq C\left(\|\mathscr{F}(z, u(q))\|_{L^{2}(Q)}+\|w z\|_{L^{2}(Q)}\right) \\
& \leq C\left(\|u(q)\|_{\mathcal{S}(0, T)}\|z\|_{\mathcal{S}(0, T)}^{2}+\|w\|_{L^{2}(Q)}\|z\|_{\mathcal{S}(0, T)}\right) \\
& \leq C\|w\|_{L^{2}(Q)}^{2} .
\end{aligned}
$$

This completes the proof.

4.2. Uniqueness and Existence of an Optimal Pair. To study the existence of an optimal pair, we present the following results.

Proposition 16. The solution mapping $q \longrightarrow u(q)$ from $\mathcal{B}_{a d}$ to $\mathcal{W}(0, T)$ of Equation (53) is continuous from the weaklystar topology of $\mathcal{B}_{\text {ad }}$ to the weak topology of $\mathcal{W}(0, T)$.

Before we prove Proposition 16, we need the following compactness lemma.

Lemma 17. Let $X, Y$, and $Z$ be Banach spaces such that the embeddings $X \subset Y \subset Z$ are continuous and the embedding $X$ $C Y$ is compact. Then, a bounded set of $W^{1, \infty}(0, T ; X, Z)=$ $\left\{g \mid g \in L^{\infty}(0, T ; X), g^{\prime} \in L^{\infty}(0, T ; Z)\right\}$ is relatively compact in $C([0, T] ; Y)$.

Proof. See Simon [24].

Proof of Proposition 16. Let $q=(c, \eta) \in \mathcal{B}_{\text {ad }}$ and let $q_{n}=\left(c_{n}\right.$, $\left.\eta_{n}\right) \in B_{\text {ad }}$ be a sequence such that

$$
q_{n} \longrightarrow q \text { weakly }-\operatorname{star} \text { in } \mathcal{B}_{\text {ad }} \text { as } n \longrightarrow \infty
$$


From now on, each state $u_{n}=u\left(q_{n}\right)$ is the weak solution of

$$
\left\{\begin{array}{l}
M_{\gamma} u_{n}^{\prime \prime}+\Delta^{2} u_{n}+k * \Delta^{2} u_{n}=\left[u_{n}, v_{n}\right]+\left(c_{n}+\eta_{n}\right) u_{n}+f, \\
\Delta^{2} v_{n}=-\left[u_{n}, u_{n}\right] \text { in } Q, \\
u_{n}=\Delta u_{n}=v_{n}=\frac{\partial v_{n}}{\partial v}=0 \text { on } \Sigma, \\
u_{n}(0, x)=u_{0}(x), u_{n}^{\prime}(0, x)=u_{1}(x) \text { in } \Omega .
\end{array}\right.
$$

If we let $p_{n}=\left(u_{0}, u_{1}, f, c_{n}+\eta_{n}\right)$ and $0=(0,0,0,0)$, then from Theorem 9, we can know that $u\left(p_{n}\right)=u_{n}$ in Equation (96) and $u(0)=0$ in $\mathcal{S}(0, T)$ and deduce that the following is fulfilled:

$$
\left\|u\left(p_{n}\right)-u(0)\right\|_{\mathcal{S}(0, T)}=\left\|u_{n}\right\|_{\mathcal{S}(0, T)} \leq C\left\|\left(u_{0}, u_{1}, f, c_{n}+\eta_{n}\right)\right\|_{\mathcal{P}} .
$$

Hence, we can deduce from (97) that $u_{n}$ and $u_{n}^{\prime}$ remain in the bounded sets of $L^{\infty}(0, T ; V) \cap \mathcal{W}(0, T)$ and $L^{\infty}\left(0, T ; H_{0}^{1}\right)$, respectively. Therefore, by using Rellich's extraction theorem, we can find a subsequence of $\left\{u_{n}\right\}$, say again $\left\{u_{n}\right\}$, and find $u \in \mathcal{W}(0, T)$ such that

$$
\begin{aligned}
& u_{n} \longrightarrow u \text { weakly in } \mathcal{W}(0, T) \text { as } n \longrightarrow \infty, \\
& u_{n} \longrightarrow u \text { weakly }-\operatorname{star} \text { in } L^{\infty}(0, T ; V) \text { as } n \longrightarrow \infty \\
& u_{n}^{\prime} \longrightarrow u^{\prime} \text { weakly }-\operatorname{star} \text { in } L^{\infty}\left(0, T ; H_{0}^{1}\right) \text { as } n \longrightarrow \infty
\end{aligned}
$$

Since $V \hookrightarrow H_{0}^{1}$ is compact, we can apply Lemma 17 to (99) and (100) with $X=V$ and $Y=Z=H_{0}^{1}$ to verify that

$$
u_{n} \text { is pre }- \text { compact in } C\left([0, T] ; H_{0}^{1}\right) \text {. }
$$

Hence, we may extract a subsequence, denoted again by $\left\{u_{n}\right\}$, such that

$$
u_{n} \longrightarrow u \text { strongly in } C\left([0, T] ; H_{0}^{1}\right) \text { as } n \longrightarrow \infty \text {. }
$$

By Lemma 2, (11) and (38), we can note for almost $t \in$ $[0, T]$ that

$$
\begin{aligned}
& \left\|\left[u_{n}(t), u_{n}(t)\right]-[u(t), u(t)]\right\|_{H^{-2}} \\
& \quad=\left\|\left[u_{n}(t)-u(t), u_{n}(t)+u(t)\right]\right\|_{H^{-2}} \\
& \quad \leq C\left\|u_{n}(t)-u(t)\right\|_{H^{1}}\left\|u_{n}(t)+u(t)\right\|_{H^{2}} \\
& \quad \leq C\left(\left\|u_{n}\right\|_{\mathcal{S}(0, T)}+\|u\|_{\mathcal{S}(0, T)}\right)\left\|u_{n}-u\right\|_{C\left([0, T] ; H_{0}^{1}\right)} \\
& \quad \leq C\left\|u_{n}-u\right\|_{C\left([0, T] ; H_{0}^{1}\right)} .
\end{aligned}
$$

Therefore, by (102) and (103), we may extract a subsequence, denoted again by $\left\{\left[u_{n}, u_{n}\right]\right\}$, such that

$$
\left[u_{n}, u_{n}\right] \longrightarrow[u, u] \text { strongly in } C\left([0, T] ; H^{-2}\right) \text { as } n \longrightarrow \infty \text {. }
$$

Since $G \in \mathscr{L}\left(H^{-2}, H_{0}^{2}\right)$, we can deduce with (104) that $v_{n}\left(=-G\left[u_{n}, u_{n}\right]\right) \longrightarrow v(=-G[u, u])$ strongly in $C\left([0, T] ; H_{0}^{2}\right)$

as $n \longrightarrow \infty$. For any $\phi \in V$, we consider for almost all $t$ $\in[0, T]$ that

$$
\begin{aligned}
& \left|\left(\left[u_{n}(t), v_{n}(t)\right]-[u(t), v(t)], \phi\right)_{2}\right| \\
& \quad \leq\left|\left(\left[u_{n}(t)-u(t), v_{n}(t)\right], \phi\right)_{2}\right|+\left|\left(\left[u(t), v_{n}(t)-v(t)\right], \phi\right)_{2}\right| .
\end{aligned}
$$

By Lemma 1, we note that the right hand side of (106) equals

$\left|\left(u_{n}(t)-u(t),\left[v_{n}(t), \phi\right]\right)_{2}\right|+\left|\left(v_{n}(t)-v(t),[u(t), \phi]\right)_{2}\right|$.

By considering $u(t), \phi \in V$, we have $[u(t), \phi] \in L^{1}$. And through (14) we can know that

$$
H_{0}^{2} \hookrightarrow L^{\infty}
$$

and therefore

$$
L^{1} \hookrightarrow H^{-2}
$$

Thus, by Lemma 2, (109), (11), and (38), we can have as follows:

right hand side of (4.54)

$$
\begin{aligned}
\leq & C\left\|u_{n}(t)\right\|_{H^{2}}^{2}\|\phi\|_{H^{2}}\left\|u_{n}(t)-u(t)\right\|_{2} \\
& +\left\|v_{n}(t)-v(t)\right\|_{H_{0}^{2}}\|[u(t), \phi]\|_{H^{-2}} \\
\leq & C\left(\left\|u_{n}\right\|_{C([0, T] ; V)}^{2}\|\Delta \phi\|_{2}\left\|u_{n}(t)-u(t)\right\|_{2}\right. \\
& \left.+\left\|v_{n}(t)-v(t)\right\|_{H_{0}^{2}}\|u\|_{C\left([0, T] ; H_{0}^{1}\right)}\|\Delta \phi\|_{2}\right) \\
\leq & C\left(\left\|u_{n}\right\|_{\mathcal{S}(0, T)}^{2}+\|u\|_{\mathcal{S}(0, T)}\right)\left(\left\|u_{n}(t)-u(t)\right\|_{2}\right. \\
& \left.+\left\|v_{n}(t)-v(t)\right\|_{H_{0}^{2}}\right)\|\Delta \phi\|_{2} \\
\leq & C\left(\left\|u_{n}-u\right\|_{C\left([0, T] ; L^{2}\right)}+\left\|v_{n}-v\right\|_{C\left([0, T] ; H_{0}^{2}\right)}\right)\|\Delta \phi\|_{2} .
\end{aligned}
$$

Thus, from (102), (105), and (110), we may extract a subsequence, if necessary, denoted again by $\left\{\left[u_{n}, v_{n}\right]\right\}$, such that

$$
\left[u_{n}, v_{n}\right] \longrightarrow[u, v] \text { strongly in } C\left([0, T] ; V^{\prime}\right) \text { as } n \longrightarrow \infty \text {. }
$$

From (95) and (101), we can obtain

$$
\left(c_{n}+\eta_{n}\right) u_{n} \longrightarrow(c+\eta) u \text { weakly in } L^{2}(Q) \text { as } n \longrightarrow \infty
$$

We replace $u_{n}$ by $u_{n_{k}}$ in Equation (96), if necessary, and take $k \longrightarrow \infty$. Then, by the standard arguments in Dautray 
and Lions ([22], pp.561-565), we conclude that the limit $y$ is a weak solution of

$$
\left\{\begin{array}{l}
M_{\gamma} u^{\prime \prime}+\Delta^{2} u+k * \Delta^{2} u=[u, v]+(c+\eta) u+f \\
\Delta^{2} v=-[u, u] \text { in } Q \\
u=\Delta u=v=\frac{\partial v}{\partial v}=0 \text { on } \Sigma \\
u(0, x)=u_{0}(x), u^{\prime}(0, x)=u_{1}(x) \text { in } \Omega
\end{array}\right.
$$

Moreover, from the uniqueness of the weak solutions, we conclude that $u=u(q)$ in $\mathcal{W}(0, T)$, which implies that $u\left(q_{n}\right) \longrightarrow u(q)$ weakly in $\mathcal{W}(0, T)$.

This completes the proof.

We now study the uniqueness and existence of an optimal pair.

Theorem 18. For sufficiently large $\alpha$ and $\beta$ in (54) there exists unique $\left(c^{*}, \eta^{*}\right) \in \mathcal{B}_{\text {ad }}$ such that $\left(c^{*}, \eta^{*}\right)$ satisfies (55).

We prove this Theorem by showing:

(i) For sufficiently large $\alpha$ and $\beta$ in (54), we prove the maps $c \longrightarrow J(c, \eta)$ and $\eta \longrightarrow J(c, \eta)$ are strictly convex and strictly concave, respectively

(ii) We prove the existence of an optimal pair by showing the maps $c \longrightarrow J(c, \eta)$ and $\eta \longrightarrow J(c, \eta)$ are lower semicontinuous and upper semicontinuous, respectively

Proof. (i) Let $\mathcal{P}_{\eta}$ be the map $c \longrightarrow J(c, \eta)$ and let $\mathcal{Q}_{c}$ be the map $\eta \longrightarrow J(c, \eta)$. To obtain the unique existence of an opti- mal pair in the minimax optimal control problem, we show the map $\mathcal{P}_{\eta}$ is strictly convex and lower semicontinuous for all $\eta \in \mathcal{D}_{\text {ad }}$ and the map $\mathcal{Q}_{c}$ is strictly concave and upper semicontinuous for all $c \in \mathcal{C}_{\text {ad }}$. As in [11] (cf. [12]), to show the strict convexity and the strict concavity of each map, we verify the following second Gâteaux derivative conditions

$$
D^{2} \mathcal{P}_{\eta}\left(c_{1}+\xi\left(c_{2}-c_{1}\right)\right)\left(c_{2}-c_{1}, c_{2}-c_{1}\right)>0, \forall c_{1}(\neq) c_{2} \in \mathcal{C}_{\mathrm{ad}},
$$

$D^{2} \mathcal{Q}_{c}\left(\eta_{1}+\xi\left(\eta_{2}-\eta_{1}\right)\right)\left(\eta_{2}-\eta_{1}, \eta_{2}-\eta_{1}\right)<0, \forall \eta_{1}(\neq) \eta_{2} \in \mathcal{D}_{\text {ad }}$,

where $0<\xi<1$. For sufficiently large $\alpha$ in (54), we first prove the convexity of $\mathcal{P}_{\eta}$ by showing (114). For simplicity, we denote $u\left(c_{1}+\xi\left(c_{2}-c_{1}\right)\right), z\left(c_{1}+\xi\left(c_{2}-c_{1}\right)\right)$ and $\rho\left(c_{1}+\xi(\right.$ $\left.\left.c_{2}-c_{1}\right)\right)$ by $u(\xi), z(\xi)$, and $\rho(\xi)$, respectively. We calculate

$$
\begin{aligned}
D \mathcal{P}_{\eta}\left(c_{1}+\xi\left(c_{2}-c_{1}\right)\right)\left(c_{2}-c_{1}\right) \\
=\lim _{l \longrightarrow 0} \frac{J\left(c_{1}+(\xi+l)\left(c_{2}-c_{1}\right), \eta\right)-J\left(c_{1}+\xi\left(c_{2}-c_{1}\right), \eta\right)}{l} \\
=D_{c} J\left(c_{1}+\xi\left(c_{2}-c_{1}\right), \eta\right)\left(c_{2}-c_{1}\right) \\
\left.=\int_{0}^{T}\left(u(\xi)-z_{d}, z(\xi)\right)_{2} d s+\alpha \int_{0}^{T}\left(c_{1}+\xi\left(c_{2}-c_{1}\right)\right), c_{2}-c_{1}\right)_{2} d t .
\end{aligned}
$$

From Corollary 14 we know that the map $q \longrightarrow u(q)$ is twice Gâteaux differentiable at $q=c_{1}+\xi\left(c_{2}-c_{1}\right)$ in the direction $c_{2}-c_{1}$. Thus from (116), we can obtain the second Gâteaux derivative of $P_{\eta}$ as follows:

$$
\begin{aligned}
D^{2} P_{\eta}\left(c_{1}+\xi\left(c_{2}-c_{1}\right)\right)\left(c_{2}-c_{1}, c_{2}-c_{1}\right) & =\lim _{\mu \longrightarrow 0} \frac{D_{c} J\left(c_{1}+(\xi+\mu)\left(c_{2}-c_{1}\right), \eta\right)\left(c_{2}-c_{1}\right)-D_{c} J\left(c_{1}+\xi\left(c_{2}-c_{1}\right), \eta\right)\left(c_{2}-c_{1}\right)}{\mu} \\
& =\int_{0}^{T}\left(u(\xi)-z_{d}, \rho(\xi)\right)_{2} d s+\|z(\xi)\|_{L^{2}(Q)}^{2}+\alpha\left\|c_{2}-c_{1}\right\|_{L^{2}(Q)}^{2},
\end{aligned}
$$

where $\rho(\xi)$ is the weak solution of Equation (75) in which $q$ and $w$ are replaced by $c_{1}+\xi\left(c_{2}-c_{1}\right)$ and $c_{2}-c_{1}$, respectively. Then, by Lemma 15 and (117), we can deduce that

$$
\begin{aligned}
& D^{2} \mathcal{P}_{\eta}\left(c_{1}+\xi\left(c_{2}-c_{1}\right)\right)\left(c_{2}-c_{1}, c_{2}-c_{1}\right) \\
& \geq-\left.\|\rho(\xi)\|_{C\left([0, T] ; L^{2}\right.}\right) \int_{0}^{T}\left\|u(\xi)-z_{d}\right\|_{2} \mathrm{ds} \\
&+\|z(\xi)\|_{L^{2}(Q)}^{2}+\alpha\left\|c_{2}-c_{1}\right\|_{L^{2}(Q)}^{2} \\
& \geq-C \sqrt{T}\|\rho(\xi)\|_{\mathcal{S}(0, T)}\left\|u(\xi)-z_{d}\right\|_{L^{2}(Q)} \\
&+\|z(\xi)\|_{L^{2}(Q)}^{2}+\alpha\left\|c_{2}-c_{1}\right\|_{L^{2}(Q)}^{2} \\
& \geq\left(\alpha-C \sqrt{T}\left\|u(\xi)-z_{d}\right\|_{L^{2}(Q)}\right) \\
& \cdot\left\|c_{2}-c_{1}\right\|_{L^{2}(Q)}^{2}+\|z(\xi)\|_{L^{2}(Q)}^{2}
\end{aligned}
$$

Hence, we can verify that there exists sufficiently large $\alpha_{l}\left(T, \mathcal{P}, \mathcal{B}_{\mathrm{ad}}, z_{d}\right)>0$ such that (114) is satisfied for any $\alpha>$ $\alpha_{l}\left(T, \mathcal{P}, \mathcal{B}_{\mathrm{ad}}, z_{d}\right)$. Therefore, the map $\mathcal{P}_{\eta}$ is strictly convex for sufficiently large $\alpha>0$.

Similarly, we can show that there exist a sufficiently large $\beta_{l}\left(T, \mathcal{P}, \mathcal{B}_{\mathrm{ad}}, z_{d}\right)>0$ such that (115) is satisfied for any $\beta>\beta_{l}\left(T, \mathcal{P}, \mathcal{B}_{\mathrm{ad}}, z_{d}\right)$. This also indicates the strict concavity of $\mathcal{Q}_{c}$.

(ii) Next, we prove the existence of an optimal pair $\left(c^{*}\right.$, $\left.\eta^{*}\right) \in \mathcal{B}_{\text {ad }}$ by verifying that $P_{\eta}$ is lower semicontinuous for all $\eta \in \mathcal{D}_{\text {ad }}$ and $\mathcal{Q}_{c}$ is upper semicontinuous for all $c \in \mathcal{C}_{\text {ad }}$. Let $\left\{c_{n}\right\} \subset \mathcal{C}_{\text {ad }}$ be a minimizing sequence of $J$. Thus

$$
\liminf _{n \longrightarrow \infty} J\left(c_{n}, \eta\right)=\inf _{c \in \mathcal{C}_{\mathrm{ad}}} J(c, \eta)
$$


Since $\left\{c_{n}\right\}$ is bounded in $L^{\infty}(Q) \subset L^{2}(Q)$, we can extract a subsequence $\left\{c_{n_{k}}\right\} \subset\left\{c_{n}\right\}$ such that

$$
c_{n_{k}} \longrightarrow c^{*} \text { weakly in } L^{2}(Q) \text { as } k \longrightarrow \infty
$$

Then, by Proposition 16, we obtain

$$
u\left(c_{n_{k}}, \eta\right) \longrightarrow u\left(c^{*}, \eta\right) \text { weakly in } \mathcal{W}(0, T) \text { as } k \longrightarrow \infty
$$

$\forall \eta \in \mathcal{D}_{\text {ad }}$. Since we know from Dautray-Lions ([22], p.480) that $\mathcal{W}(0, T) \hookrightarrow C\left([0, T] ; H_{0}^{1}\right) \cap C^{1}\left([0, T] ; L^{2}\right)$ and the embedding $H_{0}^{1} \hookrightarrow L^{2}$ is compact, we can use Lemma 17 in which $X=H_{0}^{1}, Y=Z=L^{2}$ to have from (121) that the sequence $\left\{u\left(c_{n_{k}}, \eta\right)\right\}$ is relatively compact in $C\left([0, T] ; L^{2}\right)$. Thus, we can choose a subsequence of $\left\{c_{n_{k}}\right\}$, if necessary, still denoted by $\left\{c_{n_{k}}\right\}$ such that

$$
u\left(c_{n_{k}}, \eta\right) \longrightarrow u\left(c^{*}, \eta\right) \text { strongly in } C\left([0, T] ; L^{2}\right) \text { as } k \longrightarrow \infty,
$$

$\forall \eta \in \mathcal{D}_{\text {ad }}$. Therefore, we can deduce for the same subsequence $\left\{c_{n_{k}}\right\}$ given above that

$$
u\left(c_{n_{k}}, \eta\right) \longrightarrow u\left(c^{*}, \eta\right) \text { strongly in } L^{2}(Q) \text { as } k \longrightarrow \infty
$$

$\forall \eta \in \mathcal{D}_{a d}$. Since the norm is weakly lower semicontinuous, we can verify by (120) and (123) that the map $\mathcal{P}_{\eta}$ : $c \longrightarrow J(c, \eta)$ is lower semicontinuous for all $\eta \in \mathcal{D}_{\text {ad }}$. Hence, we know that

$$
J_{0}(\eta)=\liminf _{n \longrightarrow \infty} J\left(c_{n}, \eta\right) \geq J\left(c^{*}, \eta\right), \forall \eta \in \mathcal{D}_{\mathrm{ad}} .
$$

But since $J_{0}(\eta) \leq J\left(c^{*}, \eta\right)$, we have

$$
J_{0}(\eta)=J\left(c^{*}, \eta\right)=\inf _{c \in C_{\mathrm{ad}}} J(c, \eta), \forall \eta \in \mathcal{D}_{\mathrm{ad}}
$$

By similar arguments, we can prove that $\mathcal{Q}_{c}$ is upper semicontinuous for all $c \in \mathcal{C}_{\text {ad }}$. Thus, we can also know that there exists $\eta^{*} \in \mathcal{D}_{\text {ad }}$ such that

$$
J_{0}\left(\eta^{*}\right)=\sup _{\eta \in \mathcal{D}_{\mathrm{ad}}} J_{0}(\eta)
$$

From (125) and (126), we can conclude that $\left(c^{*}, \eta^{*}\right) \epsilon$ $\mathcal{B}_{\text {ad }}$ is an optimal pair for the cost (54).

This completes the proof.

Remark 19. Assuming that $T>0$ is sufficiently small, instead of assuming $\alpha$ and $\beta$ are large enough, we can also obtain strict convexity and strict concavity of the maps $\mathcal{P}_{\eta}$ and $\mathcal{Q}_{c}$, respectively.

4.3. Necessary Condition of an Optimal Pair. In this subsection, we study the necessary optimality condition to be satisfied by an optimal pair of the minimax optimal control problem with the cost (54).

From Theorem 12, we know that the solution map $q \longrightarrow u(q)$ from $\mathcal{B}_{\mathrm{ad}}$ to $\mathcal{S}(0, T)$ is Fréchet differentiable at $q^{*}=\left(c^{*}, \eta^{*}\right)$, and the Fréchet derivative of $u(q)$ at $q=q^{*}$ in the direction $w=(h, l) \in\left[L^{\infty}(Q)\right]^{2}$, say $z=D u\left(q^{*}\right) w$ is a unique weak solution of the following equation:

$$
\left\{\begin{array}{l}
M_{\gamma} z^{\prime \prime}+\Delta^{2} z+\int_{0}^{t} k(t-s) \Delta^{2} z d s=\mathcal{G}\left(u\left(q^{*}\right), z\right)+\left(c^{*}+\eta^{*}\right) z+(h+l) u\left(q^{*}\right) \text { in } Q \\
z=\Delta z=0 \text { on } \Sigma \\
z(0)=0, z^{\prime}(0)=0 \text { in } \Omega
\end{array}\right.
$$

where $\mathcal{G}\left(u\left(q^{*}\right), z\right)$ is defined in (59). By Equation (127), we introduce the following adjoint equation corresponding to the cost function (54):

$$
\left\{\begin{array}{l}
M_{\gamma} p^{\prime \prime}+\Delta^{2} p+\int_{t}^{T} k(\sigma-t) \Delta^{2} p d \sigma=\mathcal{G}\left(u\left(q^{*}\right), p\right)+\left(c^{*}+\eta^{*}\right) p+u\left(q^{*}\right)-z_{d} \text { in } Q, \\
p=\Delta p=0 \text { on } \Sigma, \\
p(T)=0, p^{\prime}(T)=0 \text { in } \Omega .
\end{array}\right.
$$


Proposition 20. Equation (128) admits a unique solution $p \in \mathcal{S}(0, T)$.

Proof. Changing the variable $t$ to $T-t$ in Equation (128), we can complete the proof by referring to the results in $[4,17]$.

We now discuss the first-order optimality conditions for the minimax optimal control problem (55) for the quadratic cost function (54).

Theorem 21. If $\alpha$ and $\beta$ in the cost (54) are large enough or $T>0$ is sufficiently small, then an optimal control $c^{*} \in \mathcal{C}_{a d}$ and a disturbance $\eta^{*} \in \mathcal{D}_{\text {ad }}$, namely, an optimal pair $q^{*}=$ $\left(c^{*}, \eta^{*}\right) \in \mathcal{B}_{\text {ad }}$ satisfying (55) can be given by:

$$
\begin{aligned}
c^{*} & =\max \left\{\underline{c}, \min \left\{-\frac{u\left(q^{*}\right) p}{\alpha}, \bar{c}\right\}\right\}, \\
\eta^{*} & =\max \left\{\underline{\eta}, \min \left\{\frac{u\left(q^{*}\right) p}{\beta}, \bar{\eta}\right\}\right\},
\end{aligned}
$$

where $p$ is the weak solution of Equation (128), $\underline{c}$ and $\bar{c}$ are constants given in (51), and $\eta$ and $\bar{\eta}$ are constants given in (52).

Proof. Let $q^{*}=\left(c^{*}, \eta^{*}\right) \in \mathcal{B}_{\text {ad }}$ be an optimal pair in (55) with the cost (54) and $u\left(q^{*}\right)$ be the corresponding weak solution of Equation (53).

From Theorem 12, it is clear that the map $q=(c, \eta)$ $\longrightarrow u(q)$ is Gâteaux differentiable at $q^{*}=\left(c^{*}, \eta^{*}\right)$ in the direction $w=(h, l) \in\left[L^{\infty}(Q)\right]^{2}$ with $q^{*}+\varepsilon w \in \mathcal{B}_{\text {ad }}$ for sufficiently small $\varepsilon>0$. Indeed, we have

$\frac{u\left(q^{*}+\varepsilon w\right)-u\left(q^{*}\right)}{\varepsilon} \longrightarrow z(=z(w))$ strongly in $\mathcal{S}(0, T)$ as $\mathcal{E} \longrightarrow 0^{+}$,

where $z=D u\left(q^{*}\right) w$ is a unique weak solution of Equation (127). Therefore, by (130), we can get the Gâteaux derivative of the cost (54) at $q=q^{*}$ in the direction $w=(h, l)$ as follows:

$$
\begin{aligned}
D J & \left(c^{*}, \eta^{*}\right)(h, l) \\
& =\lim _{\varepsilon \longrightarrow 0^{+}} \frac{J\left(c^{*}+\varepsilon h, \eta^{*}+\varepsilon l\right)-J\left(c^{*}, \eta^{*}\right)}{\varepsilon} \\
& =\lim _{\varepsilon \longrightarrow 0^{+}} \frac{1}{2} \int_{0}^{T}\left(u\left(q^{*}+\varepsilon w\right)+u\left(q^{*}\right)-2 z_{d}, \frac{u\left(q^{*}+\varepsilon w\right)-u\left(q^{*}\right)}{\varepsilon}\right)_{2} d t \\
& +\lim _{\varepsilon \longrightarrow 0^{+}}\left[\frac{\alpha}{2} \int_{0}^{T}\left(2\left(c^{*}, h\right)_{2}+\varepsilon\|h\|_{2}^{2}\right) \mathrm{dt}-\frac{\beta}{2} \int_{0}^{T}\left(2\left(\eta^{*}, l\right)_{2}+\varepsilon\|l\|_{2}^{2}\right) \mathrm{dt}\right] \\
& =\int_{0}^{T}\left(u\left(q^{*}\right)-z_{d}, z\right)_{2} \mathrm{dt}+\alpha \int_{0}^{T}\left(c^{*}, h\right)_{2} \mathrm{dt}-\beta \int_{0}^{T}\left(\eta^{*}, l\right)_{2} \mathrm{dt},
\end{aligned}
$$

where $z=D u\left(q^{*}\right) w$ is a solution of Equation (127).
Before we proceed to the calculations, by making use of the fact that $G$ is a self adjoint operator, we can note by Lemma 1 and (109) that

$$
\begin{aligned}
(\mathscr{G} & \left.\left(u\left(q^{*}\right), \phi\right), \varphi\right)_{2} \\
& =\left(\left[\phi,-G\left[u\left(q^{*}\right), u\left(q^{*}\right)\right]\right], \varphi\right)_{2}+2\left(\left[u\left(q^{*}\right),-G\left[\phi, u\left(q^{*}\right)\right]\right], \varphi\right)_{2} \\
& =\left(\phi,\left[\varphi,-G\left[u\left(q^{*}\right), u\left(q^{*}\right)\right]\right]\right)_{2}+2\left\langle-G\left[\phi, u\left(q^{*}\right)\right],\left[\varphi, u\left(q^{*}\right)\right]\right\rangle_{2,-2} \\
& =\left(\phi,\left[\varphi,-G\left[u\left(q^{*}\right), u\left(q^{*}\right)\right]\right]\right)_{2}+2\left\langle\left[\phi, u\left(q^{*}\right)\right],-G\left[\varphi, u\left(q^{*}\right)\right]\right\rangle_{-2,2} \\
& =\left(\phi,\left[\varphi,-G\left[u\left(q^{*}\right), u\left(q^{*}\right)\right]\right]\right)_{2}+2\left(\phi,\left[u\left(q^{*}\right),-G\left[\varphi, u\left(q^{*}\right)\right]\right]\right)_{2} \\
& =\left(\phi, \mathscr{G}\left(u\left(q^{*}\right), \varphi\right)\right)_{2}, \forall \varphi, \phi \in V .
\end{aligned}
$$

We multiply both sides of the weak form of Equation (128) by $z$ which is a solution of Equation (127), and integrate it over $[0, T]$. Then, we have

$$
\begin{aligned}
\int_{0}^{T} & \left\langle M_{\gamma} p^{\prime \prime}, z\right\rangle_{V^{\prime}, V} \mathrm{dt}+\int_{0}^{T}(\Delta p, \Delta z)_{2} \mathrm{dt} \\
& +\int_{0}^{T}\left(\int_{t}^{T} k(\sigma-t) \Delta p d \sigma, \Delta z\right)_{2} \mathrm{dt} \\
& =\int_{0}^{T}\left(\mathscr{G}\left(u\left(q^{*}\right), p\right)+\left(c^{*}+\eta^{*}\right) p, z\right)_{2} \mathrm{dt} \\
& +\int_{0}^{T}\left(u\left(q^{*}\right)-z_{d}, z\right)_{2} \mathrm{dt} .
\end{aligned}
$$

By Fubini's theorem, we can know that

$$
\begin{aligned}
& \int_{0}^{T}\left(\int_{t}^{T} k(\sigma-t) \Delta p d \sigma, \Delta z\right)_{2} \mathrm{dt} \\
& \quad=\int_{0}^{T}\left(\int_{0}^{t} k(t-s) \Delta z d s, \Delta p\right)_{2} \mathrm{dt} \\
& \quad=\int_{0}^{T}\left\langle\int_{0}^{t} k(t-s) \Delta^{2} z \mathrm{~d} s, p\right\rangle_{V^{\prime}, V} \mathrm{dt} .
\end{aligned}
$$

From (132), (134) and terminal values of the weak solution $p$ of Equation (128), we can obtain the following by integration by parts of (133):

$$
\begin{gathered}
\int_{0}^{T}\left\langle p, M_{\gamma} z^{\prime \prime}+\Delta^{2} z+\int_{0}^{t} k(t-s) \Delta^{2} z \mathrm{dt}\right\rangle_{V, V^{\prime}} \mathrm{dt} \\
=\int_{0}^{T}\left(p, \mathscr{G}\left(u\left(q^{*}\right), z\right)+\left(c^{*}+\eta^{*}\right) z\right)_{2} \mathrm{dt} \\
\quad+\int_{0}^{T}\left(u\left(q^{*}\right)-z_{d}, z\right)_{2} \mathrm{dt} .
\end{gathered}
$$

Since $z$ is the solution of Equation (127), we can obtain the following from (135):

$$
\int_{0}^{T}\left(u\left(q^{*}\right)-z_{d}, z\right)_{2} \mathrm{dt}=\int_{0}^{T}\left((h+l) u\left(q^{*}\right), p\right)_{2} \mathrm{dt} .
$$


Therefore, we can deduce that (131) and (136) imply

$$
\begin{aligned}
D J\left(c^{*}, \eta^{*}\right)(h, l)= & \int_{0}^{T}\left(\alpha c^{*}+u\left(q^{*}\right) p, h\right)_{2} \mathrm{dt} \\
& +\int_{0}^{T}\left(-\beta \eta^{*}+u\left(q^{*}\right) p, l\right)_{2} \mathrm{dt} .
\end{aligned}
$$
know

Since $q^{*}=\left(c^{*}, \eta^{*}\right) \in \mathcal{B}_{\mathrm{ad}}$ is an optimal pair in (55), we

$$
D_{c} J\left(c^{*}, \eta^{*}\right)(h) \geq 0, D_{\eta} J\left(c^{*}, \eta^{*}\right)(l) \leq 0,(h, l) \in\left[L^{\infty}(Q)\right]^{2} .
$$
(138):

Therefore, we can obtain the following from (137) and

$$
\int_{0}^{T}\left(\alpha c^{*}+u\left(q^{*}\right) p, h\right)_{2} \mathrm{dt} \geq 0, \int_{0}^{T}\left(-\beta \eta^{*}+u\left(q^{*}\right) p, l\right)_{2} \mathrm{dt} \leq 0,
$$

where $(h, l) \in\left[L^{\infty}(Q)\right]^{2}$. By considering the signs of the variations $h$ and $l$ in (139), which depend on $c^{*}$ and $\eta^{*}$, respectively, we can deduce from (139) that

$$
\begin{aligned}
& c^{*}=\max \left\{\underline{c}, \min \left\{-\frac{u\left(q^{*}\right) p}{\alpha}, \bar{c}\right\}\right\}, \\
& \eta^{*}=\max \left\{\underline{\eta}, \min \left\{\frac{u\left(q^{*}\right) p}{\beta}, \bar{\eta}\right\}\right\} .
\end{aligned}
$$

This completes the proof.

\section{Data Availability}

No data were used to support this study.

\section{Conflicts of Interest}

The author declares that there is no conflict of interests regarding the publication of this paper.

\section{Acknowledgments}

This research was supported by the Daegu University Research Grant 2017.

\section{References}

[1] M. E. Bradley and S. Lenhart, "Bilinear optimal control of a Kirchhoff plate," Systems \& Control Letters, vol. 22, no. 1, pp. 27-38, 1994.

[2] J. M. Ball, J. E. Marsden, and M. Slemrod, "Controllability for distributed bilinear systems," SIAM Journal on Control and Optimization, vol. 20, no. 4, pp. 575-597, 1982.

[3] M. E. Bradley, S. Lenhart, and J. Yong, "Bilinear optimal control of the velocity term in a Kirchhoff plate equation," Journal of Mathematical Analysis and Applications, vol. 238, no. 2, pp. 451-467, 1999.

[4] J. Hwang, "Optimal control problems for a von Kármán system with long memory," Boundary Value Problems, vol. 2016, no. 1, 2016.
[5] J. L. Lions, Optimal Control of Systems Governed by Partial Differential Equations, Springer-Verlag, Berlin Heidelberg New York, 1971.

[6] I. Lasiecka and R. Triggiani, Control Theory for Partial Differential Equations: Continuous and Approximation Theories, I, Cambridge University Press, Cambridge, 2000.

[7] X. Li and J. Yong, Optimal Control Theory for Infinite Dimensional Systems, Birkhaüser, Boston, MA, USA, 1995.

[8] A. Belmiloudi, "Bilinear minimax control problems for a class of parabolic systems with applications to control of nuclear reactors," Journal of Mathematical Analysis and Applications, vol. 327, no. 1, pp. 620-642, 2007.

[9] V. Barbu and T. Precupanu, Convexity and Optimization in Banach Spaces, Reidel, Dordrecht, 1986.

[10] J. Hwang, "Fréchet differentiability for an extensible beam equation and its application to bilinear minimax control problems," Journal of Mathematical Analysis and Applications, vol. 474, no. 1, pp. 157-178, 2019.

[11] S.-U. Ryu, "Optimal control problems governed by some semilinear parabolic equations," Nonlinear Analysis: Theory, Methods \& Applications, vol. 56, no. 2, pp. 241-252, 2004.

[12] E. Zeidler, Nonlinear Functional Analysis and its Applications, II/B, Nonlinear Monotone Operator, Springer, Berlin, 1990.

[13] M. E. Bradley and S. Lenhart, "Bilinear spatial control of the velocity term in a Kirchhoff plate equation," Electronic Journal of Differential Equations, vol. 27, pp. 1-15, 2001.

[14] M. M. Cavalcanti, A. D. D. Cavalcanti, I. Lasiecka, and $\mathrm{X}$. Wang, "Existence and sharp decay rate estimates for a von Karman system with long memory," Nonlinear Analysis: Real World Applications, vol. 22, pp. 289-306, 2015.

[15] I. Chueshov and I. Lasiecka, "Long time dynamics of von Karman evolutions with thermal effects," Boletim da Sociedade Paranaense de Matemática, vol. 25, no. 1-2, pp. 37-54, 2007.

[16] G. Ji, "Uniform decay rates and asymptotic analysis of the von Kármán plate with nonlinear dissipation in the boundary moments," Nonlinear Analysis: Theory, Methods \& Applications, vol. 42, no. 5, pp. 835-870, 2000.

[17] J. Hwang and S. Nakagiri, "On semi-linear second order Volterra integro-differential equations in Hilbert space," Taiwanese Journal of Mathematics, vol. 12, no. 3, pp. 679-701, 2008.

[18] R. Temam, Infinite-Dimensional Dynamical Systems in Mechanics and Physics, vol. 68 of Applied Mathematical Sciences, Springer-Verlag, 2nd edition, 1997.

[19] R. A. Adams, Sobolev Spaces, Academic Press, New York, NY, USA, 1975.

[20] I. Chueshov and I. Lasiecka, Von Karman Evolution Equations. Wellposedness and Long-time Dynamics, Springer Monographs in Mathematics, Springer, New York, NY, USA, 2010.

[21] J. L. Lions and E. Magenes, Non-Homogeneous Boundary Value Problems and Applications I, II, Springer-Verlag Berlin Heidelberg New York, 1972.

[22] R. Dautray and J. L. Lions, Mathematical Analysis and Numerical Methods for Science and Technology, Vol. 5, Evolution Problems I, Springer-Verlag, 1992.

[23] L. C. Evans, Partial Differential Equations (Graduate Studies in Mathematics, Vol. 19), Amer Mathematical Society, Providence, RI, USA, 1998.

[24] J. Simon, "Compact sets in the space $L^{p}(\mathrm{O}, \mathrm{T} ; \mathrm{B})$," Annali di Matematica Pura ed Applicata, vol. 146, no. 1, pp. 65-96, 1986. 